\title{
Equidad-Inequidad de género en el profesorado de la Universidad de Guayaquil
}

\author{
Joaquín Francisco ÁLVAREZ HERNÁNDEZ, Rubén TRIGUEROS RAMOS, \\ Silvia SALINAS FALQUEZ, José Manuel AGUILAR PARRA, \\ Nadia Lizett SORIA MIRANDA e Isabel Damiana ALONSO LÓPEZ
}

\author{
Universidad de Almería, España
}

(Recibido el 29 de Octubre de 2019, Aceptado el 13 de Enero de 2020)

RESUMEN: Nuestra investigación se fundamenta sobre la comunidad universitaria, particularmente la de la Universidad de Guayaquil en Ecuador. Se pretende conocer el porcentaje, si lo hubiese, de inequidad de género entre el profesorado, así como validar un instrumento que nos permita determinar qué factores explican la equidad de género.

Se pretende determinar si existe diferencias en función del sexo, edad, estado, civil creencia religiosa en elementos de equidad de género como son: discriminación universitaria, bromas sexistas, responsabilidades en el hogar, equidad en la institución universitaria, acoso sexual, estereotipos sexistas, yautoestima.

La muestra del estudio estuvo conformada por 421 profesores de los cuales 203 son hombres y 218 son mujeres. Las edades están comprendidas entre los 20 y los 70 años personas. Para el análisis factorial exploratorio se utilizó una muestra independiente conformada por 228 profesores (124 hombres y 104 mujeres) entre los 34 y 47 años.

La mayoría del profesorado manifiesta tener unos valores elevados en cuanto a equidad de género en las nueve variables que conforman nuestro estudio.

Palabras clave: equidad de género; género; igualdad; discriminación sexista; acoso sexual; estereotipos sexistas; cuestionario de equidad de género.

\section{Gender Equity-Inequity in the Faculty of the University of Guayaquil}

\begin{abstract}
Our research is based on the university community, particularly that of the University of Guayaquil in Ecuador. It is intended to know the percentage, if any, of gender inequity among teachers, as well as to validate an instrument that allows us to determine which factors explain gender equit

The aim is to determine whether there are differences according to sex, age, state, civil belief in elements of gender equity such as: university discrimination, jokes, etc.). sexists, responsibilities in the home, equity in the university institution, sexual harassment, sexist stereotypes, and self-esteem.
\end{abstract}


The study sample consisted of 421 teachers, 203 of whom are men and 218 are women. Ages range from 20 to 70 people. An independent sample of 228 teachers (124 men and 104 women) between the ages of 34 and 47 was used for exploratory factor analysis.

Most teachers say they have high values for gender equity in the nine variables that make up our study.

Keywords: Gender Equity; Gender; Equality; Sexist Discrimination; Sexual Harassment; Sexist Stereotypes; Gender Equity Questionnaire.

Correspondencia: Joaquín Francisco Álvarez Hernández. Dirección: Calle Florencia 33, 04720 Aguadulce-Almería. E-mail: jalvarez@ual.es

\section{Introducción}

El desarrollo y avance cultural y/o social de los países latinoamericanos ha llevado de la mano la inquietud y concienciación de las diferentes poblaciones sobre el papel de la mujer en sus respectivas sociedades, creando políticas de igualdad real de oportunidades entre todas las personas, independientemente de su sexo; pretendiendo que la equidad de género desde los diferentes gobiernos estatales sea una realidad. Éste es el caso de Ecuador.

A pesar de los avances sociales y políticos alcanzados en Ecuador, de la Encuesta de relaciones familiares y violencia de género contra las mujeres (2011) ofrece datos demoledores:

La violencia de género contra las mujeres sobrepasa el $50 \%$ en todas las provincias del país, en todos los niveles de instrucción y el más alto porcentaje se da en mujeres alfabetizadas $70 \%$, seguida por mujeres sin ninguna instrucción $66,9 \%$, en educación básica $64 \%$, educación media $8 \%$, educación superior $55,6 \%$ y posgrado $52,8 \%$.

Seis de cada 10 mujeres de 15 y más años $(60,6 \%)$ declaran haber vivido una o más formas de violencia de género -física, psicológica, sexual o patrimonial-por parte de cualquier persona conocida o extraña, en los distintos ámbitos del quehacer social.

Una de cada 2 mujeres $(48,7 \%)$ ha recibido algún tipo de agresión por parte de los hombres con quienes tienen o tuvieron una relación de pareja.

Con anterioridad, en 1993, la Asamblea General de las Naciones Unidas aprobó la "Declaración sobre la eliminación de la violencia contra la mujer” (Res. A.G. 48/104, ONU, 1994). La violencia de género o violencia contra la mujer queda definida como:

Todo acto de violencia basado en el género que tiene como resultado posible o real un daño físico, sexual o psicológico, incluidas las amenazas, la coerción o la privación arbitraria de la libertad, ya sea que ocurra en la vida púbica o en la vida privada. La violencia física, sexual y psicológica en la familia, incluidos los golpes, el abuso sexual de las niñas en el hogar, la violencia relacionada con la dote, la violación por el marido, la mutilación genital y otras prácticas tradicionales que atentan contra la mujer, la violencia ejercida por personas distintas del marido y la violencia relacionada con la explotación; la violencia física, sexual y psicológica al nivel de la comunidad en general, incluidas las violaciones, los abusos sexuales, el hostigamiento y la intimidación sexual en el trabajo, en instituciones educacionales y en otros ámbitos, el tráfico de mujeres y la prostitución forzada; y la violencia física, sexual y psicológica perpetrada o tolerada por el Estado, dondequiera que ocurra. (Citado en Bosch y Ferrer, 2000, p. 15).

La definición anteriormente indicada es utilizada, a partir de entonces, como referencia para las acciones futuras con respecto a la violencia ejercida sobre el colectivo femenino, como

(C) Psy, Soc, \& Educ, 2020, Vol. 12(2) 
pueden ser Conferencias Mundiales sobre la Mujer (1975, 1980, 1985, 1995) y la Organización Mundial de la Salud (1998), entre otras (Bosch y Ferrer, op. Cit.). La Constitución de Ecuador de 2008, como se ha visto, también se hace eco de la misma. Todo ello, a su vez, parte del Artículo 7 de la Declaración Universal de los Derechos Humanos de 1948, el cual indica que "Toda persona tiene todos los derechos y libertades proclamados en esta Declaración, sin distinción alguna de raza, color, sexo, idioma, religión, opinión política o de cualquier otra índole, origen nacional o social, posición económica, nacimiento o cualquier otra condición". Sin embargo, "muchas mujeres y niñas aún se ven privadas, de forma rutinaria, del acceso igualitario a los recursos. Se les niega la capacidad de elección, se les arrebatan las oportunidades y se les limita por medio de estereotipos falsos y humillantes" (Alto Comisionado de las Naciones Unidas para los Derechos Humanos, 2017).

En 2011, en Ecuador, la "Encuesta Nacional de Relaciones Familiares y Violencia de Género contra las Mujeres" recoge diferentes definiciones de tipos de violencia, extraídas a su vez, de la "Ley contra la violencia a la mujer y la familia”, art. 4, y la “Convención Belém do Pará”. Dicha encuesta diferencia entre violencia física, psicológica, sexual y patrimonial, principalmente.

Esta realidad nos lleva a plantearnos el porqué de tal violencia y discriminación hacia la mujer. Qué es lo que subyace ante tales actitudes que afectan, como se ha mostrado, a muchas mujeres, independientemente de su lugar de origen, religión, cultura, raza y/o estatus socio-económico. A este respecto, Saltzman, J. (1992, p. 13) destaca dos cuestiones principales, dado la existencia de "un sistema de desventaja femenina (estratificación de los sexos)":

¿Cómo podemos explicar su permanencia y reproducción? ¿Cómo podemos explicar el cambio en el nivel de desigualdad entre los sexos? La respuesta general a la primera pregunta será que la reproducción de la desigualdad entre los sexos está fundamentalmente arraigada en la división del trabajo por sexos, tanto dentro como fuera de la familia y el hogar. Por lo tanto, la respuesta a la segunda pregunta hay que buscarla explorando aquellos procesos que contribuyen al cambio de la división del trabajo por sexos. [...] las oportunidades colectivas de las mujeres para elevar su estatus, con relación a los hombres de su sociedad, descansa en su acceso creciente al trabajo generador de recursos. Sin embargo, tal acceso está en su mayor parte controlado por élites que son masculinas, y cambia principalmente en respuesta a fuerzas que están fuera del control de las mujeres."

Así pues, y continuando con las palabras de Saltzman (op. Cit.), Merino y Pérez (2009) definen la "Equidad de género" como aquella que defiende "la igualdad del hombre y la mujer en el control y el uso de los bienes y servicios de la sociedad". Desde un enfoque feminista de la cuestión se considera que la igualdad real, o equidad de género, es aquella en la que todas las personas disfrutamos de los mismos derechos y obligaciones, independientemente de nuestro sexo, haciendo de la igualdad de oportunidades una obviedad en todos los aspectos de la vida, puesto que sin ese trato igualitario estaríamos hablando de desigualdad social: "una situación socioeconómica, no necesariamente vinculada con la apropiación o usurpación privada de bienes, recursos y recompensas, en un contexto de competencia y lucha" (Sílava, 2010, p. 112).

Además, cabe destacar, que la "Comisión Interamericana de Mujeres de la Organización de Estados Americanos", en 1994, apoyada por distintas organizaciones iberoamericanas, logró difundir la "Convención Interamericana para prevenir, sancionar y erradicar la violencia contra la mujer", aceptada por muchos de los países latinoamericanos de la región, incluyendo a Ecuador, 
lo que significa la implementación de leyes y políticas adecuadas a la misma (Quintana, Rosero, Pimentel, et al. 2014, p. 22), partiendo de su Constitución en 2008, la cual incluye varios artículos al respecto, principalmente recogidos en su Título II "Derechos". Estos, a su vez, sentaron las bases para incluir en su "Código Orgánico Integral Penal" recogido en el Registro Oficial n ${ }^{\circ} 180$ de 10 de febrero de 2014, delitos contra la violencia ejercida sobre la mujer desde su Artículo 100 "Explotación sexual" hasta el 176 "Discriminación", principalmente.

En esta cuestión, la comunidad universitaria, aun siendo considerada estandarte del conocimiento, tampoco está exenta de violencia sobre la mujer, como muestra Palomar (2011, p. 37) al indicar que:

Parecen sobrar evidencias acerca de que en el mundo académico por mucho que se quiera distinguir de otros ámbitos sociales por estar conformado por sujetos "ilustrados" se construye una práctica cotidiana similar a la de otros ámbitos sociales, sostenida por un saber común y una cultura de la inequidad de género.

Es por todo ello que nuestra investigación se fundamenta sobre la comunidad universitaria, particularmente la de la Universidad de Guayaquil en Ecuador. Se pretende conocer el porcentaje, si lo hubiese, de inequidad de género, haciendo nuestras las palabras de Palomar (op. Cit.). Así como validar un instrumento que nos permita determinar qué factores explican la equidad de género.

Se pretende determinar si existe diferencias en función del sexo, edad, estado civil, creencia religiosa en elementos de equidad de género como son: discriminación universitaria, bromas sexistas, responsabilidades en el hogar, equidad en la institución universitaria, acoso sexual, estereotipos sexistas, y autoestima.

Se ha querido incluir la variable independiente creencia religiosa por como señala (González, 2010) ser "uno de los ámbitos con gran influencia en la construcción de los estereotipos de género, es muy común escuchar en los discursos religiosos que la mujer debe someterse a la autoridad del varón ya sea su esposo o su padre, pero nunca se menciona el que tienen que compartir la responsabilidad en las labores del hogar o si pero muy esporádicamente"

También se ha querido incluir entre las variables dependientes la autoestima, es una variable muy estudiada conectándola con la equidad de género. "La autoestima es una dimensión de la autoidentidad marcada por todas las condiciones sociales que configuran a cada mujer y, de manera fundamental, por la condición de género" (Lagarde, M. 2000, pg 11) 


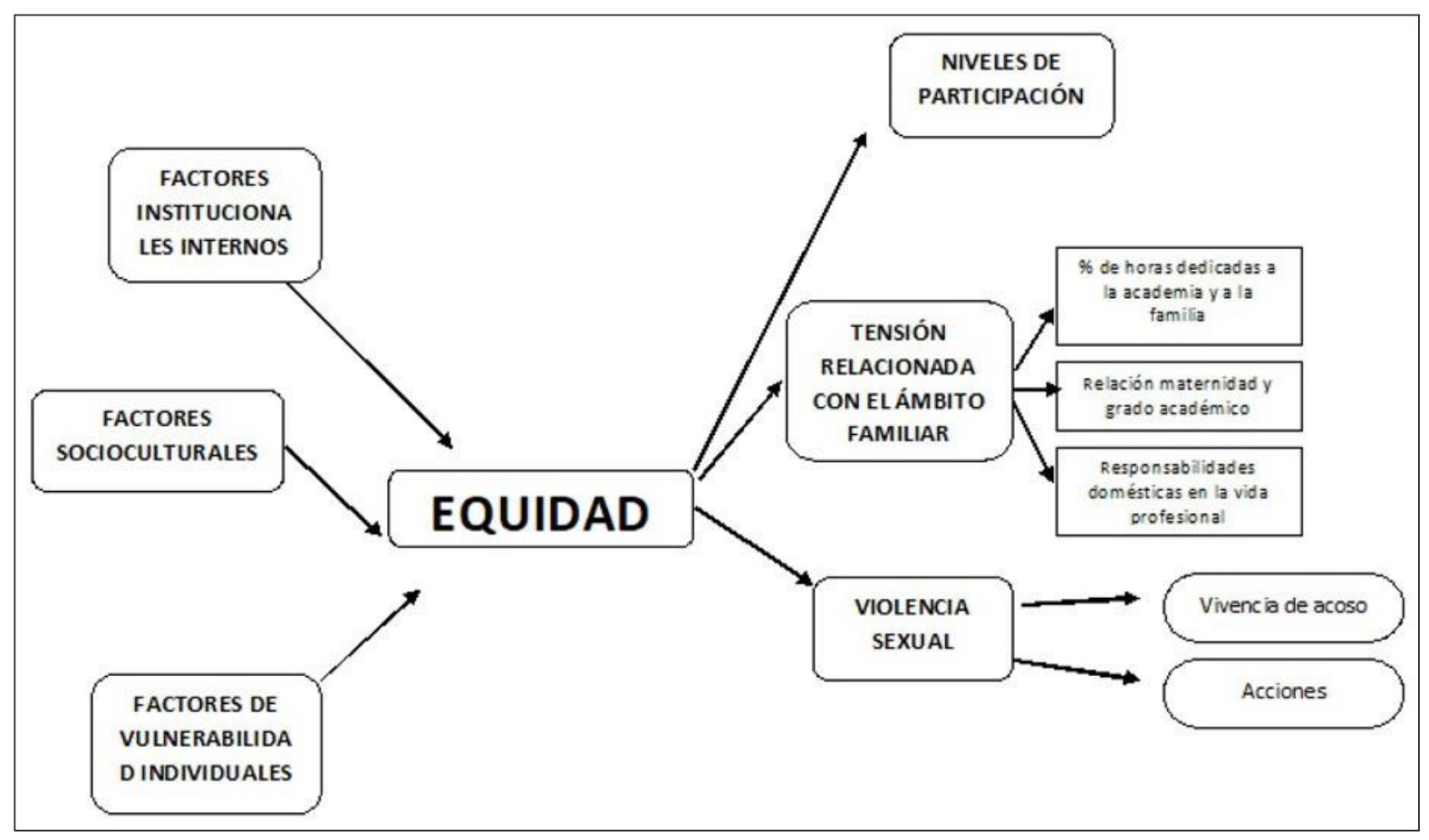

Figura 1. Modelo de factores que explican la equidad/inequidad de género y sus consecuencias

\section{Método}

\section{Participantes}

La muestra del estudio estuvo conformada por 421 profesores de los cuales 203 son hombres $(48,2 \%)$ y 218 son mujeres $51,8 \%$. Las edades están comprendidas entre los 20 y los 70 años personas, la mayoría de los encuestados están entre los 31 y los 50 años. Por porcentajes 8,3\% profesores en edades comprendidas entre los 20 y los 30 años, $33 \%$ los comprendidos entre 31 y 40 años, 28,7\% los comprendidos entre 41 y 50 años, 22,6\% los comprendidos entre 51 y 60 años y un $7,4 \%$ de profesores cuyas edades están comprendidas entre los 61 y los 70 años. El estado civil, hay 108 solteros $(25,7 \%), 250$ casados (59,4\%), 63 separados (15\%). En cuanto a las creencias religiosas la muestra se distribuye así: 262 creyentes practicantes $62.2 \%$ ), 161 creyentes no practicantes $(33.5 \%), 48$ no creyentes $(4,3 \%)$.

El muestreo empleado fue no probabilístico incidental, aunque se intentó pasar a todo el profesorado de todas las facultades de la universidad de Guayaquil la muestra que se obtuvo está en función de aquellas facultades y profesores a los que se tuvo acceso. Todos los participantes firmaron un consentimiento informado.

Para el análisis factorial exploratorio se utilizó una muestra independiente conformada por 228 profesores (124 hombres y 104 mujeres) entre los 34 y 47 años. 


\section{Procedimiento}

El estudio ha seguido todos los procedimientos éticos, respetando la custodia de los datos recopilados. Para confeccionar el cuestionario se realizaron de forma inicial seis entrevistas a profesores/as y en grupos de tres con el fin de confeccionar los ítems iniciales. Después de las entrevistas en grupos focales, también se creó una encuesta en línea para obtener información adicional (sobre los ítems resultantes) de expertos académicos, tardando 60 minutos en su cumplimentación. Para ello, se utilizó un programa de entrevista semiestructurada con el fin de facilitar su discusión general relacionada con la equidad de género. El propósito de estas entrevistas fue identificar las perspectivas de equidad que se dan entre el profesorado universitario. Los grupos de enfoque tenían una duración aproximada de 90 minutos. Los profesores/ as recibieron definiciones de equidad y se les pidió que, al considerar sus propias experiencias, comentaran la relevancia de las diversas percepciones identificadas durante la discusión en grupo. Posteriormente, se les presentó al profesorado un conjunto inicial de 54 ítems y, utilizando una escala dicotómica (aplicable vs inaplicables), se les instruyó para evaluar la relevancia de cada ítem en el contexto de la Universidad de Guayaquil, para ello, se le pidió al profesorado que clasificaran su claridad usando una escala de 7 puntos $(1=$ nada claro, $7=$ extremadamente claro). Las respuestas anónimas del profesorado fueron luego discutidas a nivel de grupo y se le pidió al profesorado la posibilidad de sugerir ítems adicionales y modificaciones de los ítems problemáticos (es decir, ítems que fueron clasificados por debajo de 4). Posteriormente, se creó un cuestionario en línea con 48 ítems y se reclutó a expertos en equidad de género, a través de un correo electrónico, para revisar el conjunto de ítems establecidos tras las entrevistas y los grupos de discusión. Los expertos utilizando una escala de 5 puntos $(1=$ pobre correspondencia a $5=$ excelente concordancia), se les pidió que indicaran en qué medida concordaban cada uno de los ítems respecto a su factor. También se les pidió que hicieran sugerencias para otros ítems alternativos o adicionales. Tras este proceso, se conformó un cuestionario final de 42 ítems, repartidos entre nueve factores con los cuales medir la equidad de género en el ámbito universitario.

\section{Instrumento}

"Cuestionario de Equidad de Género en Docentes Universitarios"

Para determinar el nivel de equidad o inequidad de género entre el profesorado de la Universidad de Guayaquil se ha elaborado una escala, que contiene 46 ítems que se organiza en nueve factores, y que se puntúan del 1 al 5 , siendo 1 "nunca" y 5 "siempre", excepto el factor siete donde 1 es "nada" y 5 es "mucho" y los factores ocho y nueve donde 1 es "totalmente en desacuerdo" y 5 "totalmente de acuerdo"

Los factores:

1. Discriminación universitaria. Pretende determinar la percepción que tienen los profesoras y profesoras, en cuanto a si existen elementos de discriminación en aspectos como reconocimientos, promoción, premios..., está conformado por 4 ítems

2. Bromas que denigran a las mujeres. Se pretende ver que percepción tienen hombres y mujeres sobre las bromas que desacreditan, desprecian a las mujeres. Está conformado por 4 ítems. 
3. Equidad vida familiar. Se pretende comprobar hasta qué punto se percibe que el desarrollo de la carrera universitaria está condicionada por el cuidado de hijos, familiares, etc. Está conformado por 4 ítems.

4. Equidad en la institución. Se trata de comprobar la percepción que tiene el profesorado en cuanto a las acciones que la universidad realiza en el ámbito de la prevención formación, investigación sobre equidad de género. Está conformado por 3 ítems

5. Acoso en puesto de trabajo. Trata de determinar si se han vivido situaciones de roces no deseados, exigencias sexuales, mensajes sexuales no deseados. Está conformado por 3 ítems.

6. Respuesta ante discriminación o acoso acciones. Se trata de comprobar cuál es la respuesta ante estas situaciones de acoso, se pide asesoramiento, se pone en manos de un abogado, se presenta una queja..., esta escala está formado por 4 ítems

7. Inacciones. Se trata de explicar por qué ante situaciones de acoso no se toman medidas. Está conformado por 6 ítems.

8. Estereotipos sexistas. Se pretende determinar la percepción que tiene el profesorado de la universidad de Guayaquil sobre algunos estereotipos como "las mujeres intentan ganar poder controlando a los hombres". Está conformado por 5 ítems

9. Autoestima. Un factor de vulnerabilidad es la autoestima se ha pretendido medir el nivel de autoestima como factor predictor de la equidad de género. Está conformado por 4 ítems.

\section{Análisis de datos}

Con el fin de determinar la validez y fiabilidad del "Cuestionario de Equidad de Género en Docentes Universitarios" en el ámbito laboral universitario se analizaron sus propiedades psicométricas. En primer lugar, se realizó un análisis factorial exploratorio (AFE). A continuación, se realizó un análisis factorial confirmatorio (AFC) con el fin de testar su estructura factorial. Posteriormente, se realizó análisis estadísticos descriptivos y se testó la fiabilidad del instrumento a través de análisis de consistencia interna (alfa de Cronbach). Además, se llevó a cabo un análisis multigrupo para analizar la invarianza respecto al género. Por último, para valorar la influencia de las variables sociodemográficas (sexo, grupo de edad, estado civil y creencia religiosa) se realizó un Mancova, prueba que permite evaluar la influencia de las mismas sobre las variables dependientes de forma conjunta y en aquellos casos en los que existió relaciones significativas se verificó el tamaño del efecto a través de la $d$ de Cohen. Para los análisis de datos se utilizaron los paquetes estadísticos SPSS 24.0 y AMOS 19.0.

Dado que el coeficiente de Mardia resultó ser alto $(1718,32)$ se utilizó el método de estimación de máxima verosimilitud junto con el procedimiento de bootstrapping para el AFC. Los estimadores no resultaron afectados por lo que se considerados robustos a pesar de la falta de normalidad (Byrne, 2001). Con el objetivo de aceptar o rechazar el modelo testado, se tuvo en consideración un conjunto de índices de ajuste: $\chi 2 / \mathrm{gl}$, CFI (Comparative Fit Index), IFI (Incremental Fit Index), RMSEA (Root Mean Square Error of Approximation) más su intervalo de confianza (IC) al 90\%, y SRMR (Standardized Root Mean Square Residual). Dado que el $\chi 2$ es muy sensible al tamaño muestral (Jöreskog y Sörbom, 1993), se empleó el $\chi 2 / g 1$, considerándose 
aceptables valores inferiores a 3 (Bentler, 1989). Los índices incrementales (CFI e IFI) muestran un buen ajuste con valores iguales o superiores 95 (Schumacker y Lomax, 1996), mientras que los índices de error (RMSEA y SRMR) se consideran aceptables con valores iguales o menores de .06 (Hu y Bentler, 1999).

\section{Resultados}

\section{Análisis factorial exploratorio}

Tabla 1. Correlaciones entre cada ítem y la puntuación total de la escala

\begin{tabular}{|c|c|c|c|}
\hline Ítems & Correlación ítem-test & $\begin{array}{l}\text { Alpha de Cronbach } \\
\text { si se elimina el ítem }\end{array}$ & $\begin{array}{l}\text { Factor de saturación } \\
\text { de cada ítem con su factor }\end{array}$ \\
\hline 1 & $.81 * *$ & .82 & $.82 \mathrm{~F} 1$ \\
\hline 2 & $.82 * *$ & .82 & $.81 \mathrm{~F} 1$ \\
\hline 3 & $.80 * *$ & .82 & $.83 \mathrm{~F} 1$ \\
\hline 4 & $.81^{* *}$ & .82 & $.84 \mathrm{~F} 1$ \\
\hline 5 & $.78^{* * *}$ & .82 & $.85 \mathrm{~F} 2$ \\
\hline 6 & $.80^{* *}$ & .82 & $.86 \mathrm{~F} 2$ \\
\hline 7 & $.76^{* *}$ & .82 & $.80 \mathrm{~F} 2$ \\
\hline 8 & $.77 * *$ & .82 & $.80 \mathrm{~F} 2$ \\
\hline 9 & $.75^{* *}$ & .82 & $.80 \mathrm{~F} 3$ \\
\hline 10 & $.79 * *$ & .82 & $.81 \mathrm{~F} 3$ \\
\hline 11 & $.83 * *$ & .82 & $.78 \mathrm{~F} 3$ \\
\hline 12 & $.70^{* * *}$ & .82 & $.77 \mathrm{~F} 3$ \\
\hline 13 & $.32 * *$ & .54 & $.31 \mathrm{~F} 3$ \\
\hline 14 & $.75^{* * *}$ & .82 & $.76 \mathrm{~F} 4$ \\
\hline 15 & $.76^{* * *}$ & .82 & $.73 \mathrm{~F} 4$ \\
\hline 16 & $.28 * *$ & .52 & $.25 \mathrm{~F} 4$ \\
\hline 17 & $.77 * *$ & .82 & $.87 \mathrm{~F} 4$ \\
\hline 18 & $.32 * *$ & .51 & $.46 \mathrm{~F} 4$ \\
\hline 19 & $.15^{* *}$ & .48 & .27 F5 \\
\hline 20 & $.72 * *$ & .82 & $.71 \mathrm{~F} 5$ \\
\hline 21 & $.74 * *$ & .82 & $.78 \mathrm{~F} 5$ \\
\hline 22 & $.17^{* * *}$ & .43 & $.24 \mathrm{~F} 5$ \\
\hline 23 & $.75^{* * *}$ & .82 & .87 F5 \\
\hline 24 & $.74 * *$ & .82 & $.75 \mathrm{~F} 6$ \\
\hline
\end{tabular}




\begin{tabular}{lccc}
\hline Ítems & Correlación ítem-test & $\begin{array}{c}\text { Alpha de Cronbach } \\
\text { si se elimina el ítem }\end{array}$ & $\begin{array}{c}\text { Factor de saturación } \\
\text { de cada ítem con su factor }\end{array}$ \\
\hline 25 & $.79 * *$ & .82 & $.81 \mathrm{~F} 6$ \\
26 & $.81^{* *}$ & .82 & $.77 \mathrm{~F} 6$ \\
27 & $.70^{* *}$ & .82 & $.79 \mathrm{~F} 6$ \\
28 & $.80^{* *}$ & .82 & $.76 \mathrm{~F} 7$ \\
29 & $.76^{* *}$ & .82 & $.73 \mathrm{~F} 7$ \\
30 & $.82^{* *}$ & .82 & $.72 \mathrm{~F} 7$ \\
31 & $.83^{* *}$ & .82 & $.83 \mathrm{~F} 7$ \\
32 & $.75^{* *}$ & .82 & $.86 \mathrm{~F} 7$ \\
33 & $.74^{* *}$ & .82 & $.77 \mathrm{~F} 7$ \\
34 & $.75^{* *}$ & .82 & $.83 \mathrm{~F} 8$ \\
35 & $.76^{* *}$ & .82 & $.82 \mathrm{~F} 8$ \\
36 & $.78^{* *}$ & .82 & $.81 \mathrm{~F} 8$ \\
37 & $.78^{* *}$ & .82 & $.86 \mathrm{~F} 8$ \\
38 & $.79 * *$ & .82 & $.82 \mathrm{~F} 8$ \\
39 & $.83^{* *}$ & .82 & $.85 \mathrm{~F} 9$ \\
40 & $.73^{* *}$ & .82 & $.84 \mathrm{~F} 9$ \\
41 & $.74^{* *}$ & .82 & $.84 \mathrm{~F} 9$ \\
42 & $.75^{* *}$ & .82 & $.79 \mathrm{~F} 9$
\end{tabular}

Nota: F1=Discriminación universitaria; F2=Bromas que denigran a las mujeres; F3=Equidad vida familiar; F4=Equidad en la institución; F5=Acoso en puesto de trabajo; F6=Respuesta ante discriminación o acoso acciones; F7=Inacciones; F8=Estereotipos sexistas; F9=Autoestima

La tabla 1 muestra las correlaciones entre cada ítem y la puntuación total de la escala que se encontraba en un rango general entre .70 y .83. Estos resultados llevan a mantener todos los ítems, puesto que la correlación ítem-test es superior al punto de corte establecido en 0.40 (Fayers y Machin, 2000). El Alpha de Cronbach global fue de .82. Además, el análisis factorial exploratorio apoya la existencia de nueve factores, mostrando un factor de saturación que osciló entre .71 y .87 .

\section{Análisis factorial confirmatorio}

Inicialmente, se evaluó la estructura del modelo de 42 ítems y nueve factores, presentando los siguientes índices de ajuste: $\chi^{2}(783, \mathrm{~N}=421)=3528.07, p=.001 ; \chi 2 / g l=4.51 ; \mathrm{CFI}=.82$; $\mathrm{TLI}=.82 ; \mathrm{IFI}=.82 ; \mathrm{RMSEA}=.091(\mathrm{IC} 90 \%=.088-.094) ; \mathrm{SRMR}=.078$. Sin embargo, tras analizar la matriz residual estandarizada covarianzas se observó la posibilidad de posibles mejoras, ya que los valores residuales de algunos ítems correlacionaban con los valores residuales de otros ítems y estaban asociados a residuales estandarizados > 2.00| (Jöreskog y Sörbom, 1993). 
De este modo, del modelo fueron excluidos un total de 5 ítems. En concreto, se eliminó dos ítems de equidad en la institución y acoso en puesto de trabajo; y un ítem de equidad vida familiar.

Excluyendo estos ítems lo índices de ajuste del modelo (Figura 1) mejoraron revelando unos índices de ajuste apropiados: $\chi^{2}(593 . N=421)=1228.47, p<.001 ; \chi^{2} / g l=2.07 ; \mathrm{CFI}=.95 ; \mathrm{TLI}=$ $.95 ; \mathrm{IFI}=.95 ; \mathrm{RMSEA}=.051(\mathrm{IC} 90 \%=.047-.055)$; SRMR $=.035$. Los pesos de regresión estandarizados oscilaron entre .62 y .98 siendo estadísticamente significativos $(p<.001)$. En cuanto a la correlación entre los factores fueron todas ellas estadísticamente significativas $(p<.001)$.

\section{Análisis de fiabilidad y descriptivos}

Tabla 2. Estadísticos descriptivos, análisis de consistencia interna y correlaciones bivaradas

\begin{tabular}{|c|c|c|c|c|c|c|c|c|c|c|c|c|}
\hline Factores & $\mathbf{M}$ & DT & $\alpha$ & 1 & 2 & 3 & 4 & 5 & 6 & 7 & 8 & 9 \\
\hline $\begin{array}{l}\text { 1. Discriminación } \\
\text { universitaria }\end{array}$ & 1.82 & .65 & .71 & & $.22 * *$ & $.69^{* *}$ & $.35^{* *}$ & $.39 * *$ & $.16^{* *}$ & $.57 * *$ & $.43^{* *}$ & $.55^{* *}$ \\
\hline $\begin{array}{l}\text { 2. Bromas que denigran } \\
\text { a las mujeres }\end{array}$ & 1.75 & .84 & .89 & & & $.33 * *$ & $.28 * *$ & $.45^{* *}$ & $.34 * *$ & $.33 * *$ & $.41 * *$ & $.14 * *$ \\
\hline 3. Equidad vida familiar & 1.48 & .64 & .72 & & & & $.50 * *$ & $.39 * *$ & $.43^{* *}$ & $.48 * *$ & $.39 * *$ & $.43^{* *}$ \\
\hline $\begin{array}{l}\text { 4. Equidad en la } \\
\text { institución }\end{array}$ & 2.42 & 1.00 & .77 & & & & & $.20^{* *}$ & $.32 * *$ & $.46^{* *}$ & $.22 * *$ & $.22 * *$ \\
\hline $\begin{array}{l}\text { 5. Acoso en puesto } \\
\text { de trabajo }\end{array}$ & 1.01 & .15 & .70 & & & & & & $.12 * *$ & $.30 * *$ & $.23 * *$ & $.36 * *$ \\
\hline $\begin{array}{l}\text { 6. Respuesta ante } \\
\text { discriminación } \\
\text { o acoso acciones }\end{array}$ & 1.06 & .34 & .93 & & & & & & & $.30 * *$ & $.50 * *$ & $.22 * *$ \\
\hline 7. Inacciones & 1.24 & .68 & .91 & & & & & & & & $.41 * *$ & $.48^{* *}$ \\
\hline 8. Estereotipos sexistas & 2.15 & .91 & .88 & & & & & & & & & $.42 * *$ \\
\hline 9. Autoestima & 4.60 & .65 & .93 & & & & & & & & & \\
\hline
\end{tabular}

** $p<.01$. Nota: $\mathrm{M}=$ Media; DT = Desviación típica; $\alpha=$ alpha de cronbach

La fiabilidad del instrumento global es de .81

La mayoría del profesorado manifiesta tener unos valores elevados en cuanto a equidad de género en las nueve variables que conforman nuestro estudio, no obstante sobre aquellas variables dependientes que han tenido un número de sujetos con unas valoraciones más dispersas como son: discriminación universitaria, bromas que denigran a las mujeres, equidad en la institución, estereotipos sexistas se pretende determinar si existe diferencias en función del sexo, edad, estado civil, creencia religiosa.

Así en lo que hace referencia al sexo, existe diferencias significativas sólo en la variable discriminación universitaria, siendo las mujeres las que puntúan más alto $(\mathrm{p}=0,048)(\mathrm{F}=3,944) \mathrm{d}$ de Cohen 0,85)

En función de la edad existen diferencias significativas en las variables discriminación universitaria $(\mathrm{p}=0,007)$, bromas que denigran a las mujeres $(\mathrm{p}=0,000)$ estereotipos sexistas se pretende $(\mathrm{p}=0,014)$ y no existe diferencias en equidad en la institución. 
Entre que edades se dan las diferencias, a través de las pruebas post hoc HSD Tuckey se ha obtenido que las diferencias se dan entre el grupo de profesores de edades comprendidas entre los (20 y 30 años) con respecto a los otros grupos (31-40 años); (41 y 50 años); (51 y 60 años) y (61-70 años)

En cuanto al estado civil, no existen diferencias significativas en ninguna de las cuatro variables.

Y finalmente en cuanto a creencia religiosa, tampoco existe diferencias significativas.

\section{Análisis de invarianza por género}

Con el fin de comprobar si la estructura factorial del modelo se muestra invariante respecto al género, se procedió a realizar un análisis multigrupo. Tal y como se muestra en la tabla 3 no se encontraron diferencias significativas entre el modelo 1 (modelo sin restricciones) y los modelos 2 (modelo de invarianza en los pesos de medida) y 3 (modelo de covarianzas estructurales invariantes). Por su parte, los resultados sí mostraron diferencias significativas entre el modelo 1 y el modelo 4 (modelo de residuos de medida invariantes). La ausencia de diferencias significativas entre el modelo 1 y el modelo 2 supone un criterio mínimo para aceptar que la estructura del modelo es invariante respecto al género (Marsh, 1993).

Tabla 3. Análisis de Invarianza por Género

\begin{tabular}{lccccccccc}
\hline Modelos & $\chi^{2}$ & $\boldsymbol{g} \boldsymbol{l}$ & $\boldsymbol{\chi}^{2} / \boldsymbol{g l}$ & $\boldsymbol{\Delta} \boldsymbol{\chi}^{2}$ & $\boldsymbol{\Delta} \boldsymbol{l}$ & $\mathbf{C F I}$ & $\mathbf{I F I}$ & RMSEA (IC 90\%) & SRMR \\
\hline Modelo 1 & 1275.95 & 593 & 2.15 & - & - & .95 & .95 & $.049(.046-.053)$ & .038 \\
Modelo 2 & 1248.02 & 600 & 2.08 & 42.67 & 21 & .95 & .95 & $.049(.045-.054)$ & .039 \\
Modelo 3 & 1208.01 & 604 & 2.00 & 51.19 & 27 & .95 & .95 & $.051(.047-.054)$ & .042 \\
Modelo 4 & 1189.27 & 612 & 1.94 & $76.56 * * *$ & 38 & .94 & .94 & $.052(.049-.055)$ & .048 \\
\hline
\end{tabular}

$* * p<.01 ; * * * p<.01$

\section{Discusión}

El objetivo del presente estudio fue analizar la validez de la estructura factorial y fiabilidad a través de la consistencia interna del cuestionario "Equidad de Género en Docentes Universitarios". Los resultados alcanzados en este estudio apoyaron que "Equidad de Género en Docentes Universitarios" como una escala que muestra una adecuada validez y fiabilidad para evaluar la equidad en el ámbito universitario de los docentes de la Universidad de Guayaquil. De esta manera, se posee una herramienta eficaz que puede ayudar a los investigadores a entender cuál es el nivel en cuanto a equidad de género en diferentes aspectos de la misma del docente universitario.

Los resultados de los AFE y AFC revelaron que la estructura factorial del cuestionario "Equidad de Género en Docentes Universitarios" poseía unos índices de ajuste adecuados para el modelo de nueve factores. Estos resultados están en sintonía con los postulados de la equidad que defiende la existencia de un conjunto de factores que se encuentran interrelacionadas entre sí y que resultan esenciales para conocer el pensamiento imperante en los individuos acerca del 
desempeño de hombre y mujeres (Osca\&López Sáez, 1994; Torres Velázquez, 2000; Gutmann, 2000; Oliva, Alfredo y Palacios (2003); Ceballos y Rodrigo, 2003; Rodríguez, Sánchez, y Torres, (2004); Páramo, Burbano y Milena, 2011)

En todos estos estudios se señalan factores explicativos de la equidad-inequidad de género, factores sociales, culturales y personales que de forma interrelacionada han ido conformando la perspectiva de género.

Los análisis de fiabilidad, a través del análisis de consistencia interna, revelaron unos índices de ajuste aceptables para las nueve subescalas. Además, el presente estudio reveló la inexistencia de una alta correlación entre los factores que integran la escala lo que demuestra la clara reciprocidad entre los factores, mostrándose en línea a estudios de Domínguez, Gutiérrez y Gómez (2017).

Aunque se pretende destacar que se han dado grandes avances con respecto a la equidad de género, la igualdad real entre hombres y mujeres, dados los cambios legislativos, culturales y/o educativos que se han llevado a cabo, lo cierto es que la realidad, investigaciones realizadas al respecto tanto a nivel nacional (Rosa Valls, Reina Capdevilla, Alejandra Montané, etc., en España) como internacional (Oswald-Spring, México; Rocío Hernández-Pozo, México; Lourdes Fernández-Rius, Cuba; Tania Pérez-Bustos, Colombia; Teresa Pedroso-Zulueta, Puerto Rico, etc.) lo desmienten. Es por ello que, desde diferentes organismos nacionales e internacionales, han asumido la falta de equidad de género o igualdad real, junto con la violencia ejercida sobre mujeres, adolescentes y niñas como un verdadero problema, convirtiéndose, en algunos aspectos, en una lacra social, buscando diferentes alternativas para su prevención y protección.

No obstante, en nuestro estudio sobre equidad de género entre el profesorado de la Universidad de Guayaquil, contradice un poco estas investigaciones y se sitúa más en la perspectiva de que, aunque la igualdad real entre hombres y mujeres aún no se ha logrado, al menos entre el profesorado de esta universidad se han dado grandes avances.

En general de todas las variables estudiadas: 1 Discriminación universitaria, 2. Bromas que denigran a las mujeres, 3. Equidad vida familiar,4. Equidad en la institución, 5. Acoso en puesto de trabajo, 6. Respuesta ante discriminación o acoso acciones, 7. Inacciones ante acoso o discriminación, 8. Estereotipos sexistas, 9. Autoestima, hemos observado que estén muy presentes entre el profesorado de la universidad de Guayaquil, excepto en autoestima, que está entre alta y muy alta.

Sólo cuatro variables tienen una presencia moderada entre el profesorado: Discriminación universitaria, Bromas que denigran a las mujeres, Equidad en la institución, Estereotipos sexistas.

En estas variables las profesoras puntúan por encima de los profesores, es decir piensan que a ellas se las discrimina más, se les hacen más bromas denigrantes, con ellas existe menos equidad en la institución y se cumplen más los estereotipos sexistas.

Así mismo, en general, son los profesores jóvenes con edades comprendidas entre los 20 y los 31 años los que observan que existe discriminación en la universidad, que se dan bromas que discriminan a las mujeres, y que se siguen utilizando los estereotipos sexistas.

\section{Fuente de financiación}

Esta investigación se enmarca dentro del Proyecto de Cooperación Internacional de la Agencia de Cooperación Internacional de la Junta de Andalucía-España, denominado "Forta- 
lecimiento participativo de la igualdad de género en la Universidad de Guayaquil y su entorno contribuyendo a la construcción de una sociedad basada en principios de justicia y equidad".

\section{Bibliografía}

Alto Comisionado para los Derechos Humanos. (2018). Conmemoración del 70 aniversario de la Declaración Universal de Derechos Humanos. https://www.europapress.es/internacional/ noticia-articulo-declaracion-derechos-humanos-derecho-igualdad-ley-20181123123553. html fecha de consulta 05/05/2019

Asamblea Constituyente. (2008). Constitución de Ecuador. Quito: Registro Oficial Órgano del Gobierno de Ecuador.

Bentler, P.(1989). EQS structural equations program manual. Los Angeles, CA: BMDP Statistical Software.

Bermúdez, M. P. (1997). La autoestima como estrategia de prevención. En G. Buela- Casal, L. Fernández y T. Carrasco, Psicología Preventiva, (pp. 105-116). Madrid: Pirámide.

Bosch, E. y Ferrer, V. (2000). Violencia de género y misoginia: Reflexiones psicosociales sobre un posible factor explicativo. Papeles del Psicólogo, 75, 13-19.

Byrne, B. M. (2001). Structural Equation Modeling with AMOS: Basic Concepts, Applications, and Programming. Mahwah, NJ: Lawrence Erlbaum Associates.

Cajas, A. K. (2011). Igualdad de género en la Constitución ecuatoriana de 2008. Revista electrónica de Derechos Humanos, 29, 1-12.

Ceballos, E. y Rodrigo, M. J. (2003). Las metas y estrategias de socialización entre padres e hijos. En M. J. Rodrigo y J. Palacios, Familia y desarrollo humano, (pp. 225-243). Madrid: Alianza Editorial.

Declaración Universal de los Derechos Humanos (1948). Artículo 7. http://www.un.org/es/ universal-declaration-human-rights/ fecha de consulta 18/05/2019

Domínguez, M., Gutiérrez, J. y Gómez, N. (2017). Equidad de género y diversidad en la educación. European Scientific Journal, ESJ (13) 7.

Fayers, P.M. and Machin, D. (2000). Quality of life: Assessment, analysis and interpretation. Chichester: John Wiley \& Sons Ltd.

González, T. (2010) Desigualdad, mujeres y religión sesgos de género en las representaciones culturales religiosas. Cuestiones de género: de la igualdad y la diferencia, N5, 467-505

Hernández-Pozo, M.R. (2013). Una mirada a la investigación actual en Latinoamérica sobre equidad y estudios de género. Acta Colombiana de Psicología, 16, 11-14. http://dx.doi. org/10.3102/00028312030004841 fecha de consulta 20/05/2019

Hu, L., \& Bentler, P. M. (1999). Cutoff criteria for fit indexes in covariance structure analysis: Conventional criteria versus new alternatives. Structural Equation Modeling”, 6, 1-55. http://dx.doi.org/10.1080/10705519909540118 fecha de consulta 20/05/2019

Instituto Ecuatoriano de Estadísticas y Censos. (2011). Primera Encuesta Nacional de Relaciones Familiares y Violencia de Género contra las Mujeres. https://anda.inec.gob.ec/anda/index. php/catalog/94 fecha de consulta 09/05/2019

Jöreskog, K. G., \& Sörbom, D. (1993). LISREL 8: Structural equation modeling with the SIMPLIS command language. Chicago: Scientific Software. 
Lagarde, M. (2000) Autoestima y Género. Cuadernos Inacabados 39.

Marsh, H. W. (1993). The multidimensional structure of academic self-concept: Invariance over gender and age. American Educational Research Journal, 30, 841-860.

Merino, M. y Pérez, J. (2009). Equidad de Género. Definición. https://definicion.de/equidad-degenero/ fecha de consulta 23/05/2019

Ministerio del Interior. (2012). Ecuador Actúa Ya. Violencia de Género, ni más. https://www. ministeriointerior.gob.ec/campana-ecuador-actua-ya-violencia-de-genero-ni-massensibiliza-a-toda-la-comunidad/\# fecha de consulta 12/05/2019

Oliva, A y Palacios, J. (2003). Familia y escuela: padres y profesores. En M. J. Rodrigo y J. Palacios, Familia y desarrollo humano, (pp. 333-350). Madrid: Alianza Editorial.

Osca A. y López-Sáez, M. (1994). Desarrollo de carrera y género. Factores que influyen en las diferencias entre hombres y mujeres. Journal of work and organizational psychology, 28, 73-86.

Palomar, C. (2011). La cultura institucional de género en la Universidad de Guadalajara. México: ANUIES.

Páramo, P., Arroyo, B y Milena, A. (2011). Género y espacialidad: análisis de actores que condicionan la equidad en el espacio público urbano. Universitas Psychologica, 10, 61-70.

Quintana, Y., Rosero, J., Pimentel, J., Camacho, G., Larrea, C. y Mendoza, C. (2014). La violencia de género contra las mujeres en el Ecuador, análisis de los resultados de la encuesta Nacional sobre relaciones Familiares y Violencia de Género contra las Mujeres. Quito: El Telégrafo.

Registro Oficial N 180 de febrero de 2014. Código Orgánico Integral Penal (COIP). https:// tbinternet.ohchr.org/Treaties/CEDAW/Shared\%20Documents/ECU/INT_CEDAW_ARL_ ECU_18950_S.pdf fecha de consulta 16/05/2019

Rodríguez, N., Sánchez, L., y Torres, L. (2004). Bajos índices académicos en educación superior: su relación con autoestima y alteraciones en la salud. En Luís A. Oblitas, Manual de psicología clínica y de la salud hospitalaria, (pp. 814-823). Bogotá: Psicom Editores.

Saltzman, J. (1992). Equidad y género. Una teoría integrada de estabilidad y cambio. Valencia: Instituto de la Mujer.

Schumacker, E. R., \& Lomax, G. R. (1996). A beginner's guide to structural equation modeling. Mahwah, NJ: Erlbaum.

Sílava, M.C. (2010). Desigualdad y Exclusión Social: De Breve Revisitación a una Síntesis Proteórica. RIPS. Revista de Investigaciones Políticas y Sociológicas, 9, 111-136.

Torres, L. E. (2011). Academic Performance, Family and Gender Equity. Ciencia y Sociedad, 36, 46-64.

Valls, R. (2008). Violencia de género en las universidades españolas. Barcelona: Ministerio de Igualdad. 


\section{APÉNDICE}

\section{CUESTIONARIO DE EQUIDAD DE GÉNERO EN DOCENTES UNIVERSITARIOS}

1. Edad:

2. Sexo: Hombre - Mujer

3. Identidad de género: Femenino - Masculino

4. Orientación sexual: Heterosexual - Bisexual - Lesbiana - Gay

5. Tiene alguna discapacidad (se considera si cuenta con carnet de discapacidad): Si No

6. Especifique el tipo de discapacidad: Auditiva - Lenguaje - Mental - Física - Otra

7. Estado civil

\begin{tabular}{|l|l|l|l|}
\hline Soltero/a & & Divorciado/a & \\
\hline Casado/a & & Separado/a & \\
\hline Unión libre & & Viudo/a & \\
\hline
\end{tabular}

8. Provincia / ciudad de origen:

9. Provincia / ciudad / Parroquia de residencia:

10. ¿Cómo se identifica usted según su cultura y sus costumbres?

\begin{tabular}{|l|l|l|l|}
\hline Indígena & Montubia & \\
\hline Afroecuatoriana/Afrodescendiente & & Mestiza & \\
\hline Negra & & Blanca & \\
\hline Mulata & & Otro ¿Cuál? & \\
\hline
\end{tabular}

11. Creencia religiosa:

Creyente prácticamente___ Creyente no practicante___ No creyente

12. Número de hijos e hijas que viven el hogar (biológicos, adoptivos y de la pareja):

13. Edad a la que tuvo su primer hijo:

14. Grado académico

Tercer nivel __ Maestría ___ Candidato a Doctorado___ Doctorado
Otro (especifique):

15. Tipo de relación laboral:

\begin{tabular}{|l|l|l|l|}
\hline Contrato & & Nombramiento & \\
\hline
\end{tabular}


16. Facultad:

\begin{tabular}{|l|l|l|l|}
\hline Arquitectura y Urbanismo & Ciencias Químicas & \\
\hline Ciencias Administrativas & Comunicación Social & \\
\hline Ciencias Agrarias & Educación Física, Deportes y Recreación & \\
\hline Ciencias Matemáticas y físicas & Filosofía, Letras y Ciencias de Educación & \\
\hline Ciencias Médicas & Ingeniería Industrial & \\
\hline Ciencias Naturales & Ingeniería Química & \\
\hline Ciencias para el Desarrollo & Jurisprudencia, Ciencias Sociales y Políticas & \\
\hline Ciencias Psicológicas & Medicina Veterinaria y Zootecnia & \\
\hline & Odontología & \\
\hline
\end{tabular}

17. Carrera:

- Especificar carrera:

18. Jornada de trabajo:

\begin{tabular}{|l|l|l|l|}
\hline Diurna & & Nocturna & \\
\hline
\end{tabular}

19. Cargo:

\begin{tabular}{|l|l|l|l|}
\hline Decano/a & & Gestor/a & \\
\hline Director/a de Carrera & & Docente sin cargo administrativo & \\
\hline Coordinador/a de la Unidad & & & \\
\hline
\end{tabular}

20. Área de trabajo:

\begin{tabular}{|l|l|l|l|}
\hline Dirección de la Facultad/Carrera & Bienestar Estudiantil & \\
\hline $\begin{array}{l}\text { Formación Académica } \\
\text { (incluye clases y prácticas pre profesionales) }\end{array}$ & Posgrado & \\
\hline Investigación & & Tutor/a de prácticas y/o trabajos de titulación & \\
\hline Gestión social del conocimiento (Vinculación) & & & \\
\hline
\end{tabular}

21. ¿Cuántas horas dedica diariamente a:

\begin{tabular}{|l|c|c|c|}
\cline { 2 - 4 } \multicolumn{1}{c|}{} & Menos de 8h & Entre 8 y 16h & Más de 16h \\
\hline Trabajo laboral & & & \\
\hline Trabajo en el cuidado del hogar & & & \\
\hline
\end{tabular}


Evalúe con qué frecuencia se dan las siguientes situaciones por ser hombre o mujer en la Universidad:

\begin{tabular}{|l|l|l|l|l|l|}
\cline { 2 - 5 } \multicolumn{1}{c|}{} & Nunca & $\begin{array}{c}\text { Rara } \\
\text { vez }\end{array}$ & $\begin{array}{c}\text { Algunas } \\
\text { veces }\end{array}$ & Frecuentemente & $\begin{array}{c}\text { Muy } \\
\text { frecuentemente }\end{array}$ \\
\hline $\begin{array}{l}\text { Recibo reconocimientos o premios por } \\
\text { mi trabajo. }\end{array}$ & & & & & \\
\hline $\begin{array}{l}\text { He sido discriminado por razones } \\
\text { de género en esta institución. }\end{array}$ & & & & & \\
\hline $\begin{array}{l}\text { Alguna vez me han excluido de alguna } \\
\text { actividad, premio, permiso o promoción. }\end{array}$ & & & & & \\
\hline $\begin{array}{l}\text { He recibido una evaluación injusta por } \\
\text { parte de algún ente evaluador. }\end{array}$ & & & & & \\
\hline
\end{tabular}

Con cuánta frecuencia escucha o presencia cada uno de los siguientes comportamientos en su salón de clase o Facultad:

\begin{tabular}{|l|l|l|l|l|l|}
\cline { 2 - 5 } \multicolumn{1}{c|}{} & Nunca & $\begin{array}{c}\text { Rara } \\
\text { vez }\end{array}$ & $\begin{array}{c}\text { Algunas } \\
\text { veces }\end{array}$ & Frecuentemente & $\begin{array}{c}\text { Muy } \\
\text { frecuentemente }\end{array}$ \\
\hline $\begin{array}{l}\text { Los Docentes (hombres) hacen bromas y } \\
\text { comentarios que estereotipan y denigran a } \\
\text { las mujeres. }\end{array}$ & & & & & \\
\hline $\begin{array}{l}\text { Los Docentes (hombres) hacen bromas y } \\
\text { comentarios que estereotipan y denigran a } \\
\text { los hombres. }\end{array}$ & & & & & \\
\hline $\begin{array}{l}\text { Las Docentes (mujeres) hacen bromas y } \\
\text { comentarios que estereotipan y denigran a } \\
\text { las mujeres. }\end{array}$ & & & & & \\
\hline $\begin{array}{l}\text { Las Docentes (mujeres) hacen bromas y } \\
\text { comentarios que estereotipan y denigran a } \\
\text { los hombres. }\end{array}$ & & & & & \\
\hline
\end{tabular}

\section{Con qué frecuencia se dan las siguientes situaciones en la vida familiar:}

\begin{tabular}{|l|l|l|l|l|l|}
\cline { 2 - 5 } \multicolumn{1}{c|}{} & Nunca & $\begin{array}{c}\text { Rara } \\
\text { vez }\end{array}$ & $\begin{array}{c}\text { Algunas } \\
\text { veces }\end{array}$ & Frecuentemente & $\begin{array}{c}\text { Muy } \\
\text { frecuentemente }\end{array}$ \\
\hline $\begin{array}{l}\text { Mi progreso académico se ha visto } \\
\text { afectado por cuidar a mis hijos e hijas. }\end{array}$ & & & & & \\
\hline $\begin{array}{l}\text { Mi progreso académico se ha visto } \\
\text { afectado por cuidar a mi pareja. }\end{array}$ & & & & & \\
\hline $\begin{array}{l}\text { Mi progreso académico se ha visto } \\
\text { afectado por cuidar a personas con } \\
\text { discapacidad. }\end{array}$ & & & & & \\
\hline $\begin{array}{l}\text { Mi progreso académico se ha visto } \\
\text { afectado por cuidar a otras personas. }\end{array}$ & & & & & \\
\hline
\end{tabular}


Con qué frecuencia se ha dado las siguientes situaciones en la institución:

\begin{tabular}{|l|l|l|l|l|l|}
\cline { 2 - 5 } \multicolumn{1}{c|}{} & Nunca & $\begin{array}{c}\text { Rara } \\
\text { vez }\end{array}$ & $\begin{array}{c}\text { Algunas } \\
\text { veces }\end{array}$ & Frecuentemente & $\begin{array}{c}\text { Muy } \\
\text { frecuentemente }\end{array}$ \\
\hline $\begin{array}{l}\text { La Universidad ha implementado } \\
\text { políticas, planes y acciones para pro- } \\
\text { mover la equidad de género. }\end{array}$ & & & & & \\
\hline $\begin{array}{l}\text { Las mallas curriculares de las carreras } \\
\text { de la Universidad cuentan con } \\
\text { asignaturas sobre género y sexualidad. }\end{array}$ & & & & & \\
\hline $\begin{array}{l}\text { La Universidad promueve la investi- } \\
\text { gación y la gestión social en temas de } \\
\text { género y equidad. }\end{array}$ & & & & & \\
\hline
\end{tabular}

Con qué frecuencia y por parte de quién Usted ha vivido las siguientes situaciones en la institución:

\begin{tabular}{|l|l|l|l|l|l|}
\cline { 2 - 5 } \multicolumn{1}{c|}{} & Nunca & $\begin{array}{l}\text { Rara } \\
\text { vez }\end{array}$ & $\begin{array}{l}\text { Algunas } \\
\text { veces }\end{array}$ & Frecuentemente & $\begin{array}{l}\text { Muy } \\
\text { frecuentemente }\end{array}$ \\
\hline $\begin{array}{l}\text { Mensajes de naturaleza sexual no } \\
\text { deseados. }\end{array}$ & & & & & \\
\hline $\begin{array}{l}\text { Exigencia a realizar actividades que } \\
\text { no competen a sus funciones por } \\
\text { rechazar proposiciones sexuales. }\end{array}$ & & & & & \\
\hline Roces o contacto físico no deseado & & & & & \\
\hline
\end{tabular}

En caso de que se hayan dado algunas de las situaciones descritas anteriormente, con qué frecuencia ha realizado las siguientes acciones:

\begin{tabular}{|l|l|l|l|l|l|}
\cline { 2 - 5 } \multicolumn{1}{c|}{} & Nunca & Rara vez & $\begin{array}{l}\text { Algunas } \\
\text { veces }\end{array}$ & frecuentemente & $\begin{array}{l}\text { Muy } \\
\text { frecuentemente }\end{array}$ \\
\hline Pidió una Investigación & & & & & \\
\hline $\begin{array}{l}\text { Pidió una asesoría en esta } \\
\text { institución }\end{array}$ & & & & & \\
\hline $\begin{array}{l}\text { Presentó una queja ante las } \\
\text { autoridades de la institución }\end{array}$ & & & & & \\
\hline Contrató un abogado & & & & & \\
\hline
\end{tabular}


En el caso de que NUNCA ha tomado ninguna acción al respecto de las situaciones mencionadas, indique en qué medida han influido las siguientes razones:

\begin{tabular}{|l|l|l|l|l|l|}
\cline { 2 - 6 } \multicolumn{1}{c|}{} & Nada & Poco & Algo & Bastante & Mucho \\
\hline Por desconocimiento & & & & & \\
\hline Por temor a represalias & & & & & \\
\hline Temor al agresor & & & & & \\
\hline Por vergüenza & & & & & \\
\hline Porque no confía en que se hará algo & & & & & \\
\hline Temía dañar su reputación & & & & & \\
\hline
\end{tabular}

A continuación, se presentan una serie de frases sobre los hombres y las mujeres y sobre su relación mutua en nuestra sociedad contemporánea. Indique en que medida USTED PIENSA ASÍ:

\begin{tabular}{|l|l|l|l|l|l|}
\cline { 2 - 5 } \multicolumn{1}{c|}{} & $\begin{array}{c}\text { Totalmente en } \\
\text { desacuerdo }\end{array}$ & $\begin{array}{c}\text { En } \\
\text { desacuerdo }\end{array}$ & $\begin{array}{c}\text { Ni de } \\
\text { acuerdo ni en } \\
\text { desacuerdo }\end{array}$ & $\begin{array}{c}\text { De } \\
\text { acuerdo }\end{array}$ & $\begin{array}{c}\text { Totalmente } \\
\text { de acuerdo }\end{array}$ \\
\hline $\begin{array}{l}\text { Las mujeres intentan ganar po- } \\
\text { der controlando a los hombres. }\end{array}$ & & & & \\
\hline $\begin{array}{l}\text { Las mujeres exageran los } \\
\text { problemas que tienen en el } \\
\text { trabajo. }\end{array}$ & & & & \\
\hline $\begin{array}{l}\text { Una vez qua una mujer logra } \\
\text { que un hombre se comprometa } \\
\text { con ella, por lo general intenta } \\
\text { controlarle estrechamente. }\end{array}$ & & & & \\
\hline $\begin{array}{l}\text { Cuando las mujeres son ven- } \\
\text { cidas por los hombres en una } \\
\text { competencia justa, general- } \\
\text { mente ellas se quejan de haber } \\
\text { sido discriminadas. }\end{array}$ & & & & & \\
\hline $\begin{array}{l}\text { Existen muchas mujeres que } \\
\text { para burlarse de los hombres, } \\
\text { primero se insinúan sexual- } \\
\text { mente a ellos y luego rechazan } \\
\text { los avances de éstos. }\end{array}$ & & & & & \\
\hline
\end{tabular}


Evalúe el sentimiento de satisfacción que tiene de sí misma. Conteste las frases con la respuesta que considere más apropiada.

\begin{tabular}{|c|c|c|c|c|c|}
\hline & $\begin{array}{c}\text { Muy en } \\
\text { desacuerdo }\end{array}$ & $\begin{array}{c}\text { En } \\
\text { desacuerdo }\end{array}$ & $\begin{array}{c}\text { Ni de acuerdo } \\
\text { ni en } \\
\text { desacuerdo }\end{array}$ & $\begin{array}{c}\text { De } \\
\text { acuerdo }\end{array}$ & $\begin{array}{l}\text { Muy de } \\
\text { acuerdo }\end{array}$ \\
\hline $\begin{array}{l}\text { Estoy convencido de que tengo } \\
\text { cualidades buenas. }\end{array}$ & & & & & \\
\hline $\begin{array}{l}\text { Soy capaz de hacer las cosas tan } \\
\text { bien como la mayoría de la gente. }\end{array}$ & & & & & \\
\hline $\begin{array}{l}\text { Tengo una actitud positiva hacia } \\
\text { mí misma/o. }\end{array}$ & & & & & \\
\hline $\begin{array}{l}\text { En general estoy satisfecha/o de } \\
\text { misma/o. }\end{array}$ & & & & & \\
\hline
\end{tabular}

